\title{
دراسة اقتصادية للوضع المائى الراهن فى مصر
}

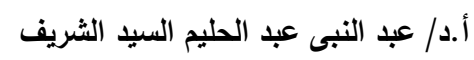

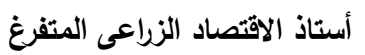

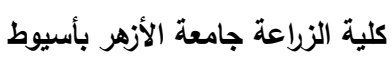

أ.د/ خيرى طه إبراهيم

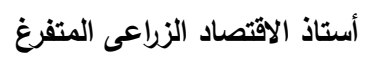

كلية الزراعة جامعة المنيا

\author{
أبو رحاب عصمت فؤاد أبو رحاب

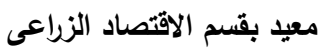 \\ كلية الزراعة . جامعة الأزهر بأسيوط الزاعط
}

تعد دراســـة الوضـــع الراهن للموارد المائية القومية والاســــــامات من الأهمية بمكان للتعرف على مدى إمكانية تغطية تلك الموارد المائية الحالية

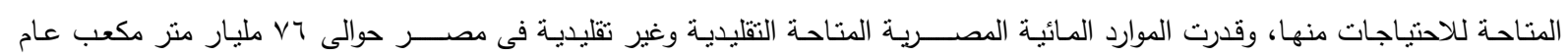

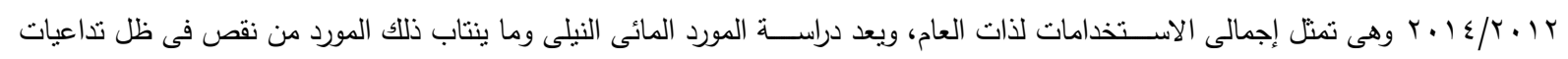

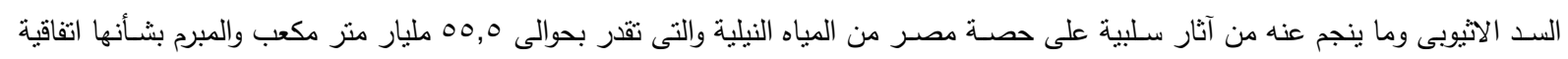

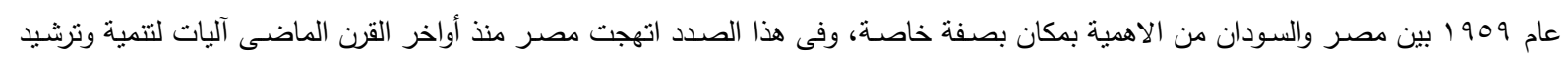

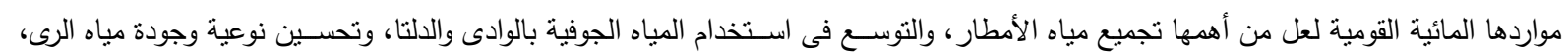

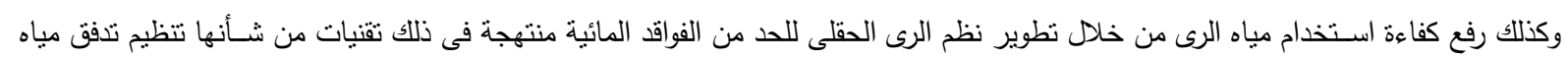

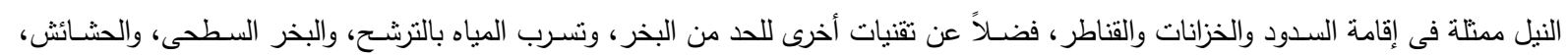
والثوازنات، التسرب الأرضى، والفقد من خلال فروع التوزيع والحقل وتوليد الكهرباء والسدة الثنتوية.

مشكلة البحث: يعتبر النقص المضطرد فى كميات المياه المتاحة على المستوى القومى من مختلف مصادرها، وتزايد الاستخدامات المختلفة منها

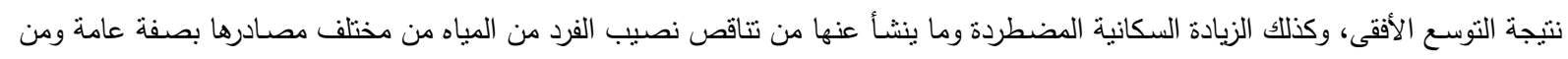

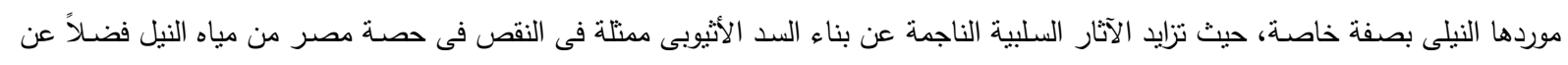

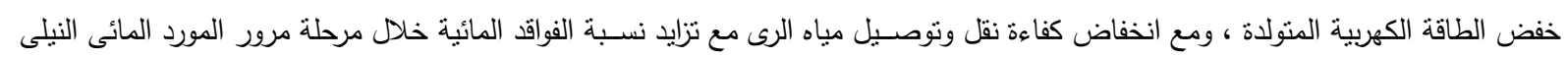

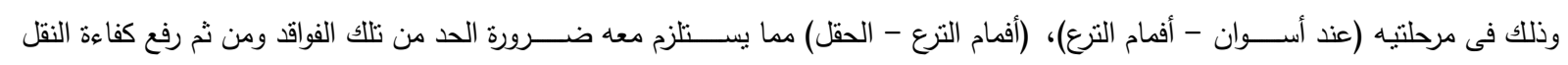
والتوصيل للمياه النيلية.

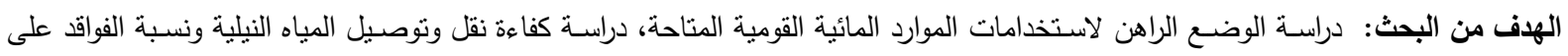

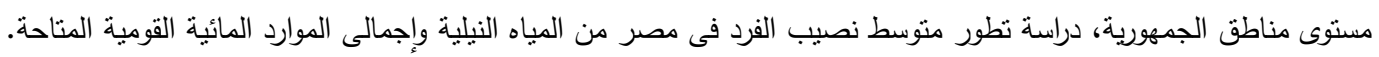

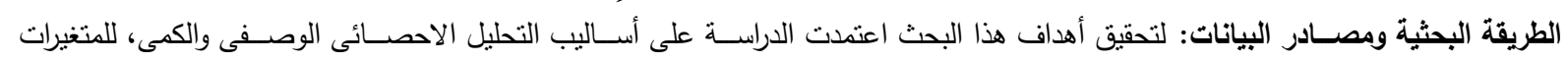

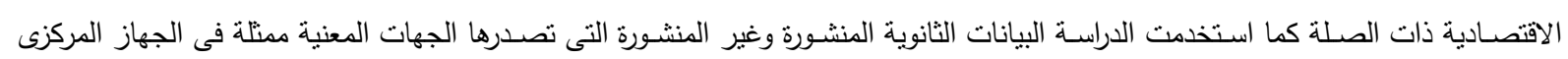

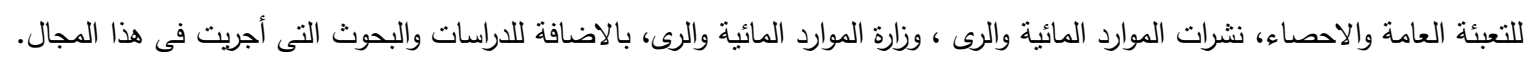

ثانياً: الموارد المائية المتاحة واستخدامتها:

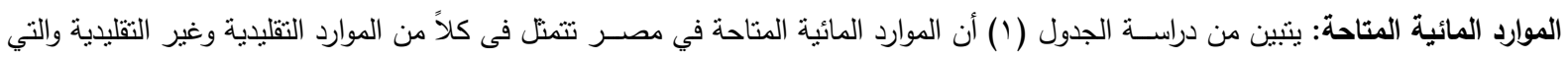

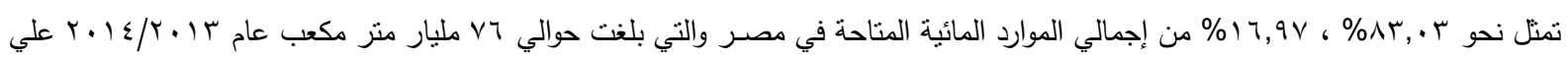

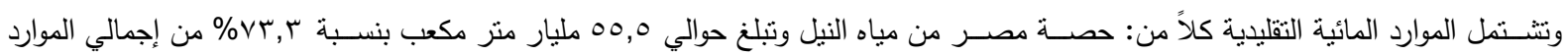

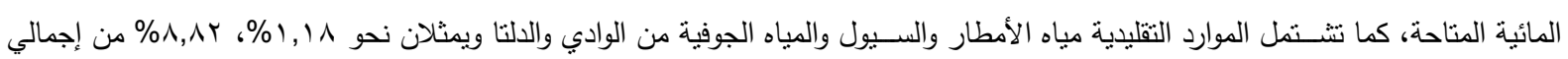

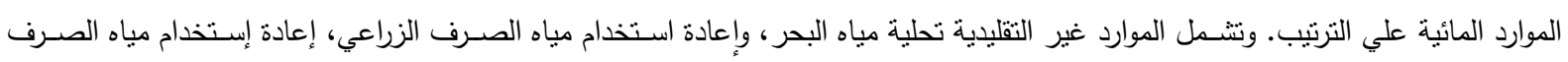

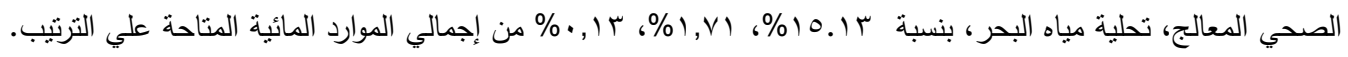




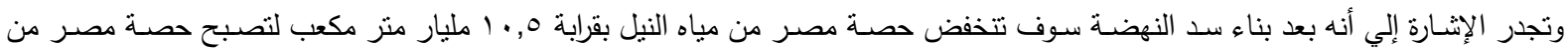

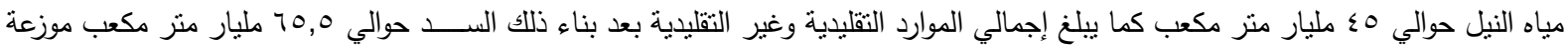

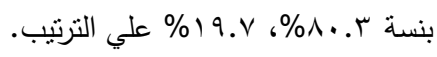
الاستخدامات المائية: يتبين من دراسة الجدول (Y) أن الاستخدامات المائية تتمنل في احتياجات الزراعة من الموارد المتاحة، الفاقد بالبخر من نهر

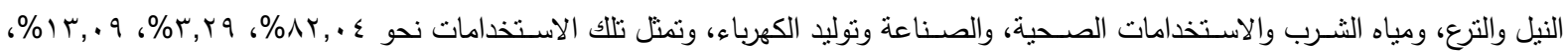

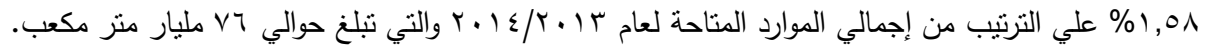

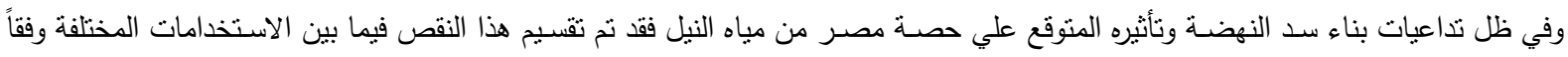

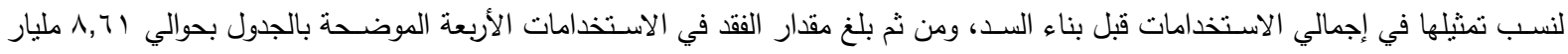

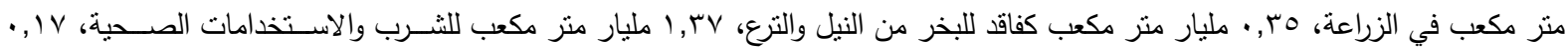

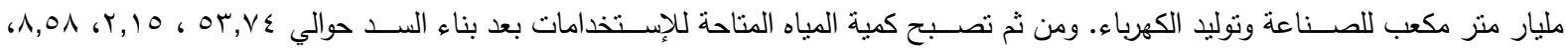

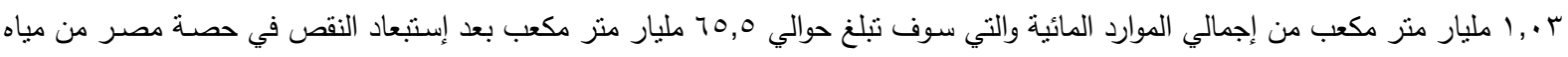
النيل بعد بناء السد.

\begin{tabular}{|c|c|c|c|c|c|}
\hline \multicolumn{2}{|c|}{ \% من الإجمالي } & \multicolumn{2}{|c|}{ الكمية المتاحة من المياه } & \multirow[t]{2}{*}{ مصدر المياه } & \multirow{2}{*}{ نوع الموارد } \\
\hline بعد بناء السد & قالأثبوبي السد & بعد بناء السد الأثيوبي & قالأثيوبي السد & & \\
\hline $7 \wedge, \vee \cdot$ & $V T, \cdot r$ & $\leqslant 0$ & 00,0 & نهر النيل (حصة مصر من مياه النيل) & تقليدية \\
\hline $1, r v$ & 1,11 & $\cdot, 9$ & $\cdot, 9$ & مياه الأمطار و السيول & \\
\hline $1 \cdot, Y T$ & $\wedge, \wedge r$ & $7, \mathrm{~V}$ & $7, V$ & المياه الجوفية من الو ادي و الدلتا & \\
\hline$\Lambda \cdot, r$ & $\Delta r, r$ & or,, 1 & $\pi r, 1$ & إجمالي الموارد التقليدية & \\
\hline $1 V, 07$ & 10,11 & 11,0 & 11,0 & إعادة إستخدام مياه الصرف الزر اعي & \\
\hline 1,99 & $1, \sqrt{ } 1$ & $1, r$ & $1, r$ & إعادة إستخدام مياه الصرف الصحي المعالج & \\
\hline$\cdot, 10$ & $\cdot, 1 T$ & $\cdot, 1$ & $\cdot, 1$ & تحلية مياه البحر & \\
\hline $19, V \cdot$ & $17,9 V$ & $1 r, 9$ & $1 r, 9$ & إجمالي الموارد غير التقليدية & \\
\hline$\% 1 \cdots$ & $\% 1 \cdots$ & 70.0 & $V 7$ & & الإجمالي الكلي \\
\hline
\end{tabular}

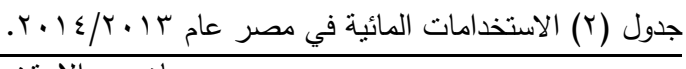

\begin{tabular}{|c|c|c|c|c|c|}
\hline 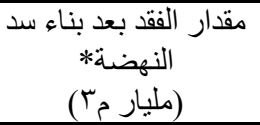 & 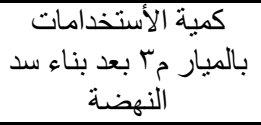 & 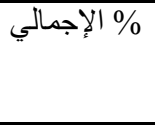 & الاستخدامات مليار مَ) & بيان & الاستخدمات \\
\hline$\wedge, 71$ & or, $\mathrm{V \varepsilon}$ & $\% \wedge r, \cdot \varepsilon$ & Tr, Mo & & 1- الزراعة \\
\hline$\cdot, r_{0}$ & $r, 10$ & $\%$ \%, Yq & $r, 0$ & & rـ الفاقد بالبخر من النيل و الترع \\
\hline $1, r v$ & $1,0 \wedge$ & $\% 1 T, \cdot 9$ & 9,90 & & rـ الثرب و الإسخدامات الصحية \\
\hline$\cdot, 1 \mathrm{~V}$ & $1, \cdot r$ & $\% 1,0 \wedge$ & $1, r$ & & ــ الصناعة وتوليد الكهرباء وأخري \\
\hline- & - & - & - & & 0ـ الملاحة النهرية \\
\hline $1 \cdot, 0$ & 70,0 & $\% 1 \ldots$ & $\mathrm{V7}$ & & الإجمالي \\
\hline
\end{tabular}

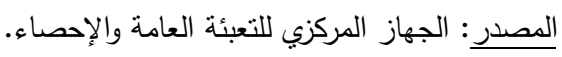

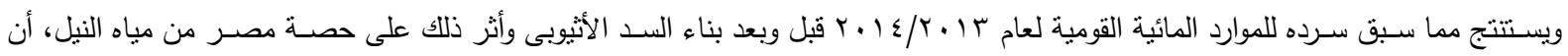

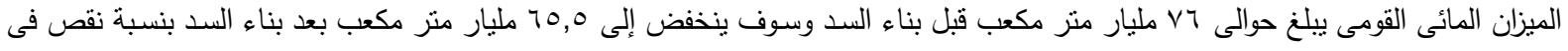

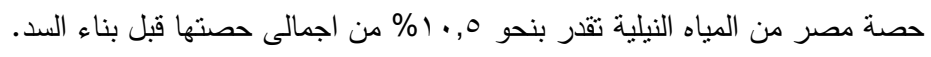


ثالثاً: متوسط كمية مياه الري والفاقد منها خلال المورد المائى النيلى:

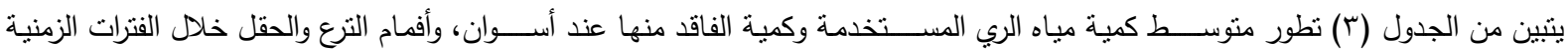

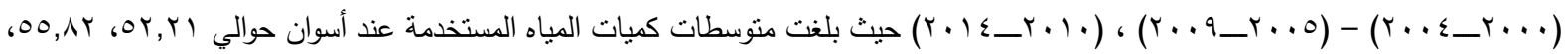

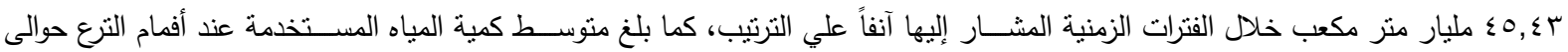

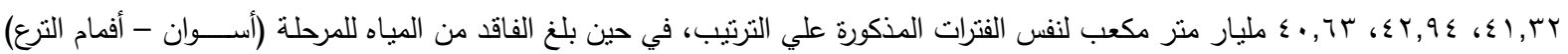

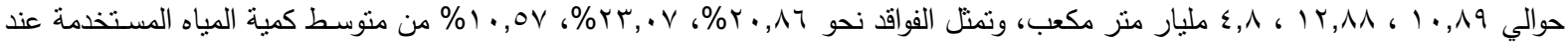

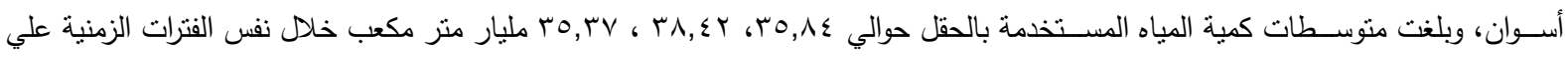

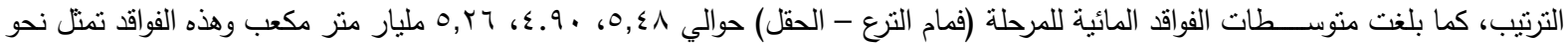

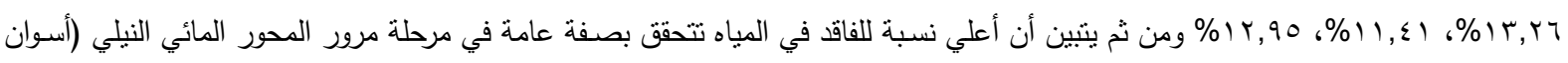

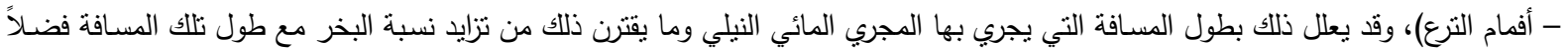
عن الاستخدامات غير الزراعية لمياه النيل خلال نلاك المرحلة.

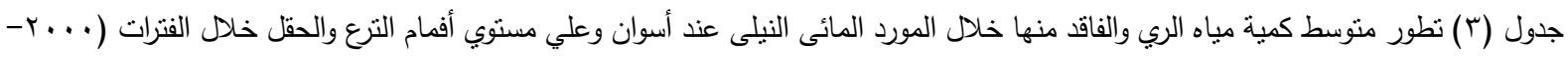

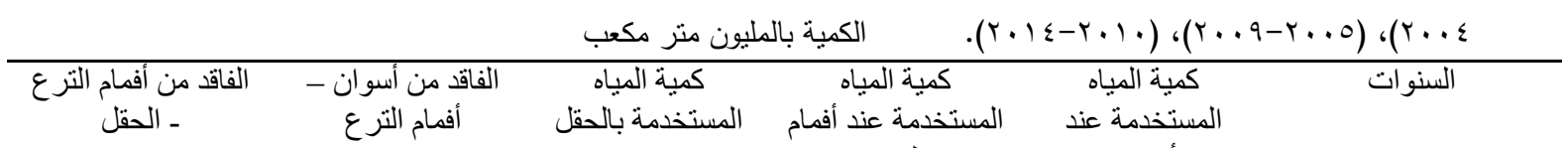

\begin{tabular}{|c|c|c|c|c|c|}
\hline & & & الراع & 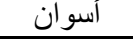 & \\
\hline 019 & 1.771 & 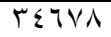 & 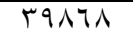 & 0.047 & r... \\
\hline$O Y \leqslant Y$ & T.YIY & rEVOV & r9999 & $0 . Y 11$ & $r \ldots 1$ \\
\hline $0 Y 90$ & $1.91 \varepsilon$ & rorvt & $\varepsilon .77 \Lambda$ & $010 \lambda r$ & T.. T \\
\hline $091 \leqslant$ & 1119. & rroor & $\varepsilon Y \leqslant T T$ & 04704 & $r \ldots r$ \\
\hline$O V \leqslant T$ & $11 \leqslant \leqslant r$ & $r \vee 100$ & $\varepsilon r 09 \lambda$ & $00 . \varepsilon_{0}$ & $T \cdots \varepsilon$ \\
\hline $0 \leqslant \vee 7.1$ & $1 \cdot 110 Y$ & rONET & $\varepsilon 1719.1$ & Orr.o & 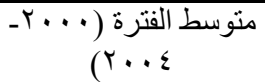 \\
\hline 0774 & 1.797 & ravVo & 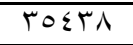 & $\varepsilon \pi \mid r \varepsilon$ & $r \ldots o$ \\
\hline TाTo & $1771 \varepsilon$ & $\varepsilon .9 \leqslant 1$ & $\varepsilon r \cdot \lambda r$ & $0979 \mathrm{~V}$ & $T \cdots T$ \\
\hline 7.77 & $1 \times 99 \varepsilon$ & $\varepsilon Y \cdot V_{0}$ & $\{\Lambda 1 \leqslant 1$ & $711 \% 0$ & $T \cdots V$ \\
\hline 7.17 & ITrE纟 & $\varepsilon Y \wedge \leq T$ & $\sum \wedge \Lambda 0 Y$ & $7 Y .97$ & $r \cdots \wedge$ \\
\hline ETYT & I.NTY & $r \leqslant 071$ & rq)AV & 0.119 & $r \ldots q$ \\
\hline$\varepsilon \wedge 99 . r$ & TrAVT & 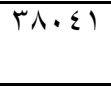 & $\varepsilon r q \varepsilon \cdot . r$ & 00117.4 & 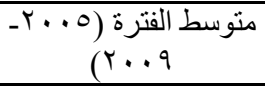 \\
\hline$\sum \wedge 94$ & $101 \mathrm{~V}$ & rVYqE & EYTAV & $01 Y \cdot \varepsilon$ & $r \cdot 1$. \\
\hline $7.9 Y$ & $7 \times 70$ & $\Gamma \cdot \Lambda T Y$ & 47909 & ETYYL & $r .11$ \\
\hline$\{119$ & TrI. & $r r 1.9$ & TY9YA & $\varepsilon .1 \Gamma \Lambda$ & $T \cdot I T$ \\
\hline $0 r \cdot 1$ & $r \ldots 7$ & rVAIV & $\varepsilon r \cdot r_{0}$ & $\sum \because . \mu 1$ & $T \cdot 1 T$ \\
\hline orl. & r99r & rArOA & $\varepsilon r 07 \Lambda$ & $\leqslant 707$. & $r \cdot 1 \leqslant$ \\
\hline OYTE. & $\sum \vee 9 \lambda$ & rorra & $\varepsilon .7 r T . \varepsilon$ & $\varepsilon 0 \leqslant \pi) . \varepsilon$ & 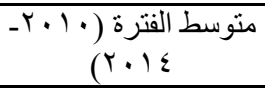 \\
\hline
\end{tabular}

رابعاً: المعالم الاتجاهية المقدرة للكميات المستخدمة خلال مراحل المورد المائى النيلى:

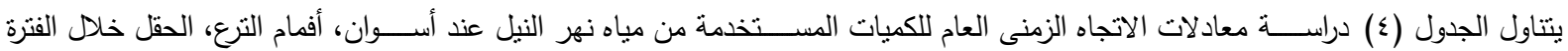

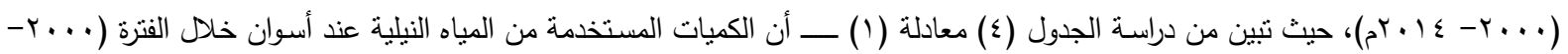

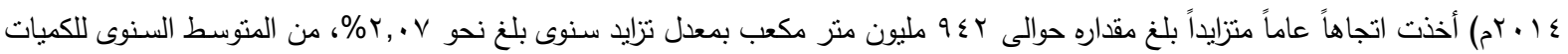

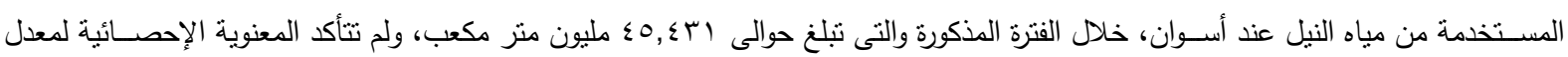

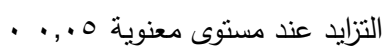

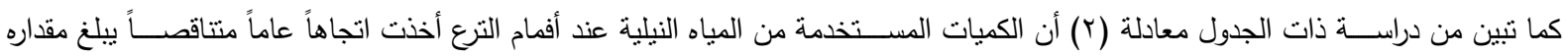

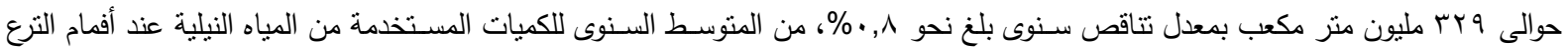




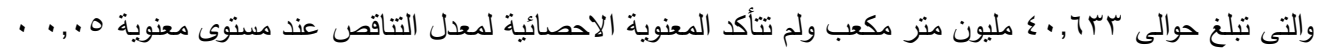

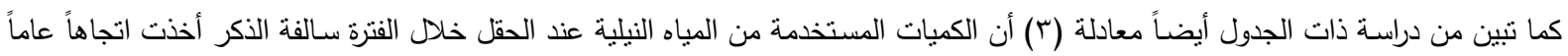

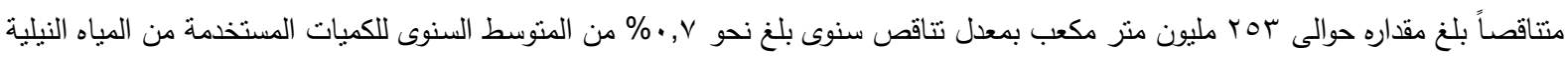

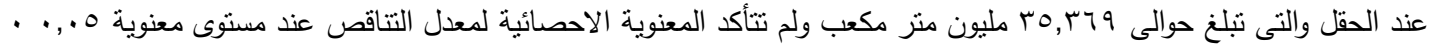

\begin{tabular}{|c|c|c|c|c|c|c|c|c|}
\hline المعادلة & التغنير & التغير & قيمة " & $\begin{array}{l}\text { التحديدل } \\
\text { R2 }\end{array}$ & نموذج الدالة & أوفق ألمقرة & متوسط المتغير & المعالم \\
\hline$(1-r)$ & $r . \cdot V$ & $9 \leqslant Y$ & $r . V T$ & 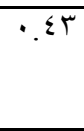 & $\begin{array}{l}\mathrm{y}_{1}=4387+4421 \mathrm{X}-478 \mathrm{X}^{2}+ \\
11.9 \mathrm{X}^{3} \\
\quad(114) \quad(-.86) \quad(0.52)\end{array}$ & تكعيبية & $\leqslant 0, \varepsilon \Gamma$ & أمستخدمة المياه عند \\
\hline$(Y-Y)$ & $(\cdot \wedge)$ & $(r r q)$ &.${ }^{4}$ & $\because 9$ & $\begin{array}{l}\mathrm{y}_{2}=36236+2634 \mathrm{X}-322 \mathrm{X}^{2} \\
+11.4 \mathrm{X}^{3} \\
(0.9) \quad(-.77) \quad(0.66)\end{array}$ & تكعيبية & $\varepsilon \cdot, \tau$ & أمستخدمة المياه عند الترماه \\
\hline$(r-r)$ & $(\cdot V)$ & $(Y \circ Y)$ &.$r q$ & $\because 1$ & $\begin{array}{l}\mathrm{y}_{3}=30818+2672 \mathrm{X}- \\
319 \mathrm{X}^{2}+11.1 \mathrm{X}^{3} \\
\quad(0.91) \quad(-.67) \quad(0.64)\end{array}$ & تكعيبية & ro,rV & المستخدمة عية المياه \\
\hline
\end{tabular}

تشير y1 ، y2 y3 ،لكميات التقديرية من مياه الرى المستخدمة عند أسوان ، أفمام التزع، والحقل

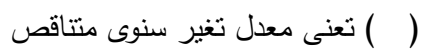

المصدر: جدول رقم (r).

خامساً: كفاءة نقل وتوصيل المياه:

أ. على مستوى مناطق الجمهورية:

تقدر الاحتياجات الإروائية للزروع النباتية بالعروات المختلفة علي أسـاس المقنن الحقلي يضـاف إلى إليه الفواقد من الحقل في أفمام الترع وكذلك الفواقد

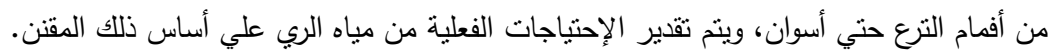

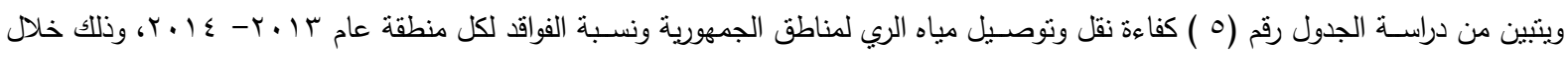
مرحلتي التوصـيل الذي يمر به المورد المائي (أسـوان - أفمام التزع)، (أفام الترع - الحقل)، فبالنسبة للمرحلة (أسـوان - أفمام الترع) تبين انخفاض

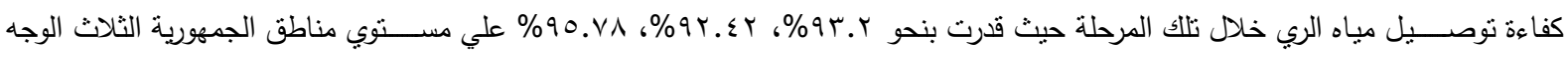

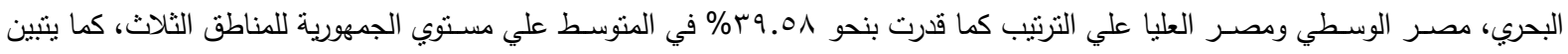

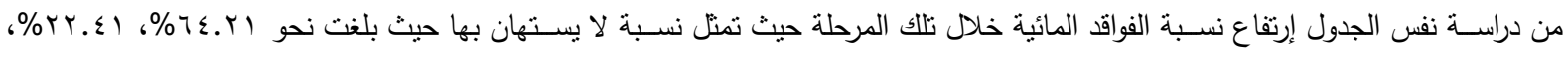

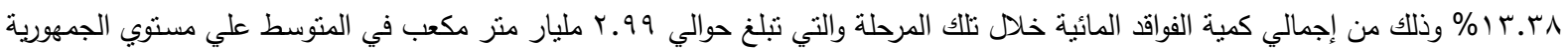

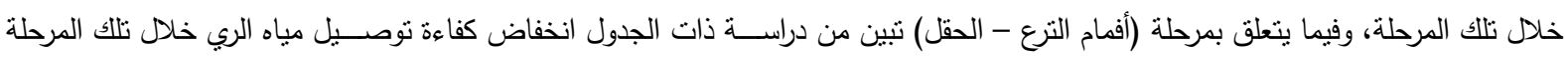

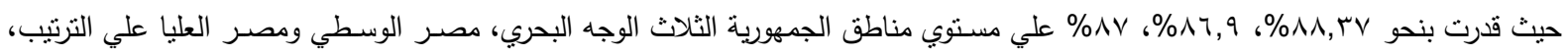

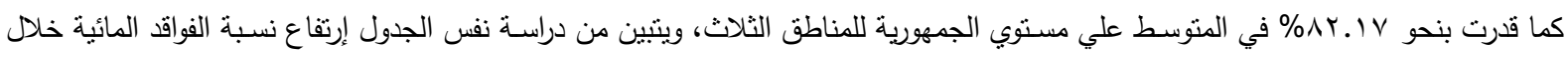

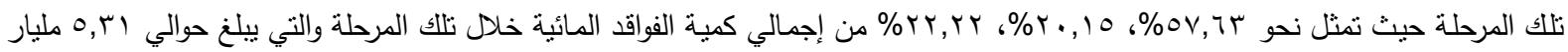
متز مكعب في المتوسط علي مستوي الجمهورية. 


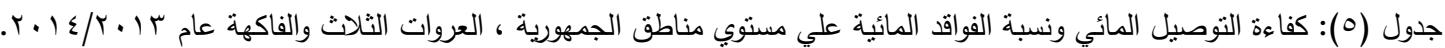

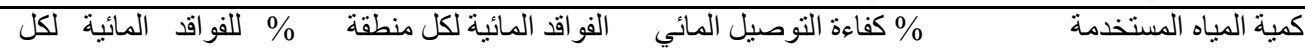
منطقة

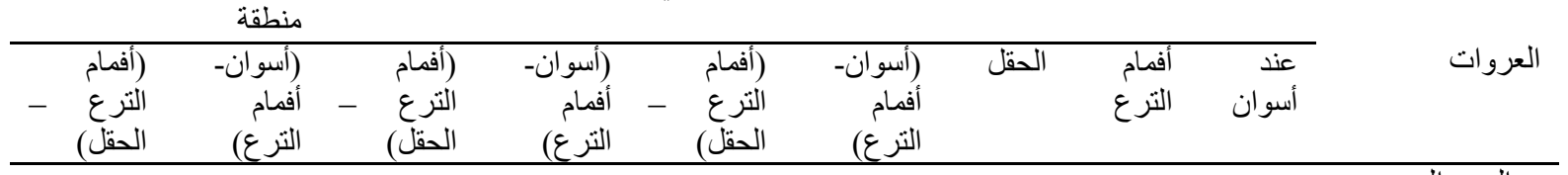

\begin{tabular}{|c|c|c|c|c|c|c|c|c|c|}
\hline & & & & & & & & & الوجه البحري \\
\hline$r \varepsilon .7 \varepsilon$ & $r 4 . \leqslant 0$ & 1.7 & $\because V$ & 97.94 & 94.0 & $V .0$ & 1.11 & $\wedge . \wedge 1$ & شتوي \\
\hline $07 . Y 1$ & $0 \leq .1 \mathrm{~V}$ & $1 . V Y$ & $1 . \cdot \varepsilon$ & N9.r & 94.94 & $1 \leq . \Gamma \wedge$ & 17.1. & $1 V .1 \leq$ & صيفي \\
\hline Y.9ะ & $r .1 T$ & $\because 9$ & $\because 7$ & 人५. $\vee 7$ & 91.19 & .09 & $\cdot .71$ & $\cdot V \varepsilon$ & نيلي \\
\hline $7 . Y 1$ & 7.10 & .19 & $.1 T$ & 人.. & QY.YT & $1 . Y \varepsilon$ & $1 . \leqslant r$ & 1.00 & فاكهة \\
\hline$\% 1 \ldots$ & $\% 1 \ldots$ & $r .9$ & 1.94 & $\wedge \wedge . \leqslant 1$ & $94.1 \mathrm{~V}$ & TY.YT & YT.YY & $T \wedge . T \varepsilon$ & نالي الوجه \\
\hline
\end{tabular}

\begin{tabular}{|c|c|c|c|c|c|c|c|c|c|}
\hline & & & & & & & & & مصر الوسطي \\
\hline 10.01 & TV.rT &.$r \Lambda$ &. .10 & $\wedge 7 . \wedge 0$ & $9 T_{.} \cdot \varepsilon$ & r.01 & r.^9 & r.1ร & شتوب \\
\hline$\sum 9.04$ & $\left.\sum V . V\right\urcorner$ & .04 & $\cdot \mu^{\mu}$ & $\wedge \uparrow . \wedge \Lambda$ & 94.77 & $r .01$ & $\varepsilon . \varepsilon$ & ร.น & صيفي \\
\hline$V . \leqslant \Lambda$ & $V . \leqslant 7$ & $\because \cdot 1$ & $\because .0$ & AV.79 & 94.10 & $.0 \mathrm{~V}$ & .70 & $\cdot V \cdot$ & نيلي \\
\hline$\vee . \Sigma \Lambda$ & $V . \leqslant 7$ & $\because \cdot 1$ &. .0 & $\wedge 7 . \leq \leqslant$ & 94.19 & .01 & .09 & $.7 \varepsilon$ & فاكهة \\
\hline \multirow[t]{2}{*}{$\% 1 \ldots$} & $\% 1 \ldots$ & $1 . V$ & $.7 V$ & งร. 9. & $9 Y . \leqslant Y$ & V.1 & A.IV & $\Lambda . \wedge \varepsilon$ & إجمالي مصر \\
\hline & & & & & & & & & مصر العليًا \\
\hline$r 7 . r V$ & ro. &.$M^{\prime \prime}$ & .1 & $\wedge 7 . \wedge 7$ & 90.94 & $r .0$ & T.r. & T.ST & شتوي \\
\hline 77.90 & $7 V .0$ & .189 & $\cdot r V$ & $\Lambda V .10$ & 90.19 & 0.4 & 7.10 & $T . \varepsilon Y$ & صيفي \\
\hline 1.79 & r.o & $\because \cdot Y$ & $\because \cdot 1$ & $\wedge 1 . \wedge 1$ & $91.7 \mathrm{~V}$ & $\because .9$ & .11 &. $.1 Y$ & نيلي \\
\hline 0.9 & 0 & $\because \cdot 7$ & $\because \cdot r$ & 17.97 & $90 . \wedge r$ & $\because \varepsilon \cdot$ &.$\leqslant 7$ & $\cdot . \leqslant \wedge$ & فاكهة \\
\hline \multirow[t]{2}{*}{$\% 1 \ldots$} & $\% 1 \ldots$ & 1.11 &.$\varepsilon$ & $\Lambda V_{.} .$. & $90 . V \wedge$ & V.9. & 9.91 & $9 . \leqslant 1$ & إجمالي مصر العليا \\
\hline & & & & & & & & & الجمهورية \\
\hline rY.VV & ro. $\leqslant 0$ & $1 . V \leqslant$ & 1.9 & $97.9 V$ & $94.7 \varepsilon$ & 11.71 & 11.40 & $1 \leq . \leq 1$ & شتوي \\
\hline OV.7T & $0 \leqslant .11$ & $r .7$ & 1.74 & AN.rV & qE. . & Tr.TE & r..r. & rV.qT & صيفي \\
\hline r.OV & $\varepsilon .+1$ &. .19 &. .14 & $\wedge \uparrow . \wedge 1$ & $9 Y . M 1$ & 1.50 & $1 . \leqslant \varepsilon$ & 1.07 & نيلي \\
\hline $7 . \cdot r$ & 7.47 & 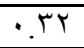 &. .19 & $9 \vee .1$. & $9 r . \wedge \wedge$ & Y.17 & $r . \varepsilon \Lambda$ & Y.TV & فاكهة \\
\hline$\% 1 \ldots$ & $\% 1 \ldots$ & 0.4 & r.99 & $\wedge \vee . \wedge 1$ & 94.01 & rA.rT & $\varepsilon r .0 V$ & $\sum 7.07$ & إجمالي الجمهورية \\
\hline
\end{tabular}

ب . على مستوى العروات الثلاث: يتتاول هذا الجزء دراسة كفاءة التوصيل المائي ونسبة الفواقد المائية خلال مرحلتي مرور المورد المائي النبلي (أسوان - أفمام التزع)، (أفمام الترع -

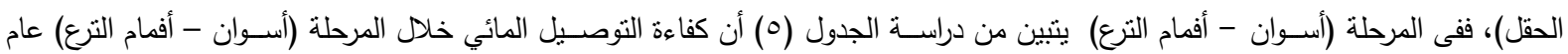

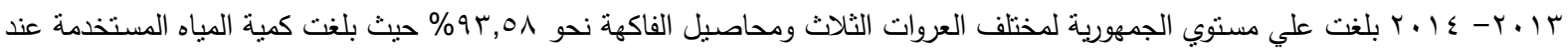

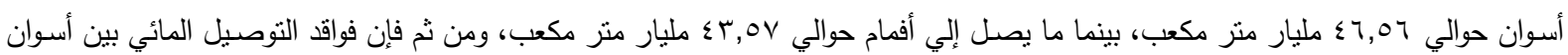

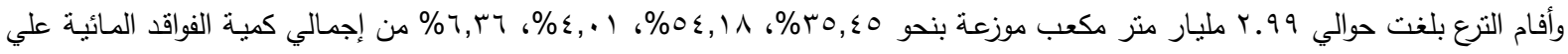

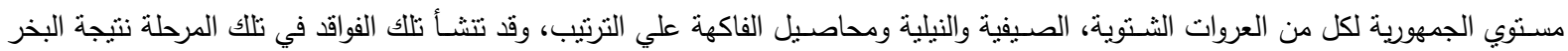
من أسطح المجاري المائية والتسرب فضلاً عن وجود خلل بشبكات التوصيل المائي التي تمتد لمسافات طويلة .

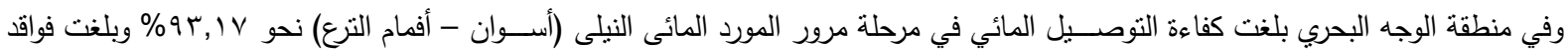

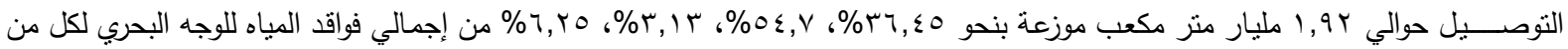
العروات الثـتوية، والصـيفية، والنيلية، ومحاصيل الفاكهة علي الترتيب، وفي منطقة مصـر الوسطي بلغت كفاءة النتوصيل المائي النيلى فى مرحلة

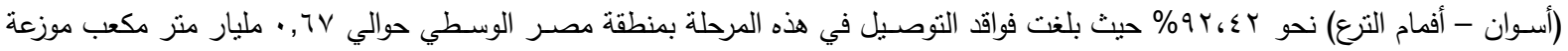

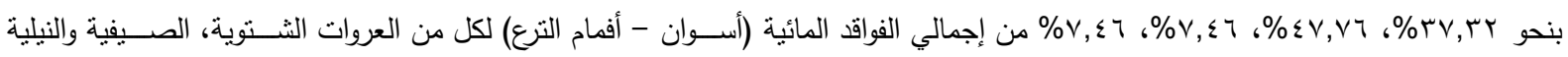

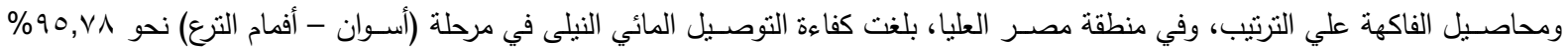

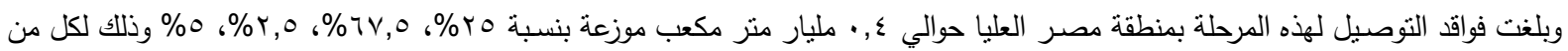
العروات الثتوية، والصيفية، والنيلية، ومحاصيل الفاكهة علي الترتيب. 


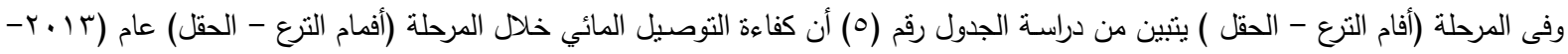

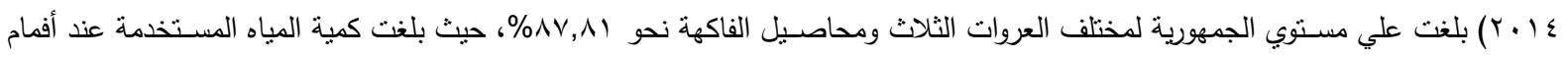

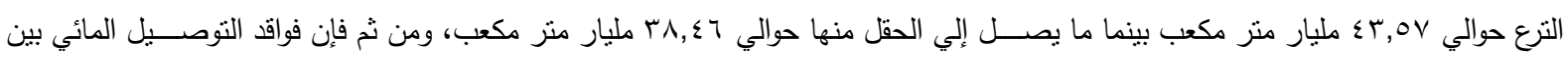

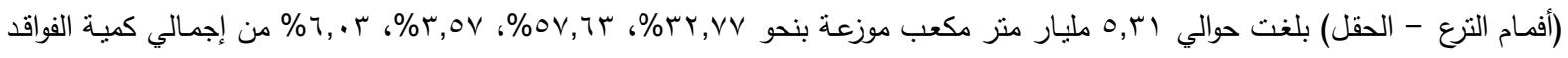

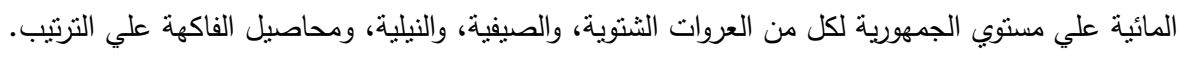

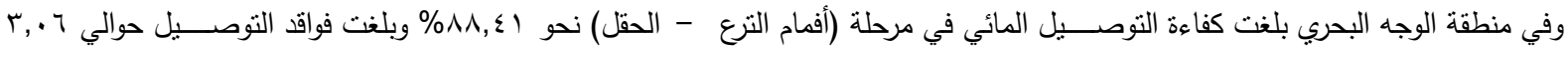

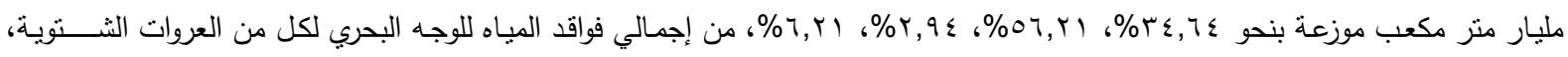
والصيفية، والنيلية، ومحاصيل الفاكهة علي الترتيب، وفي منطقة مصر الوسطي بلغت كفاءة التوصيل المائي فى مرحلة (أفمام التزع - الحقل) نحو التحو

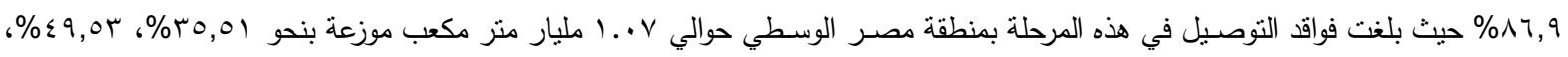

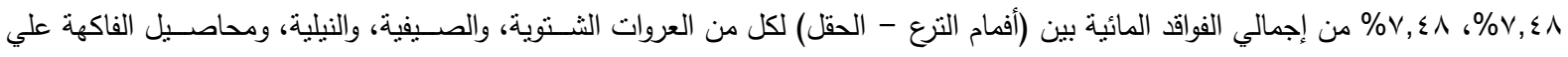

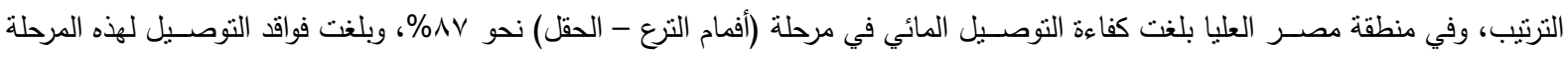

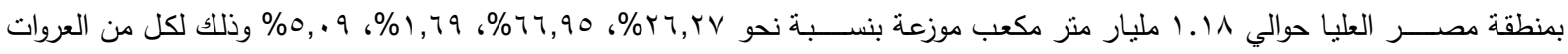
الثنوية، والصيفية والنيلية ومحاصيل الفاكهة علي الترتيب.

سادساً: تطور متوسط نصيب الفرد من المياه النيلية ومن الموارد المائية الإجمالية المتاحة:

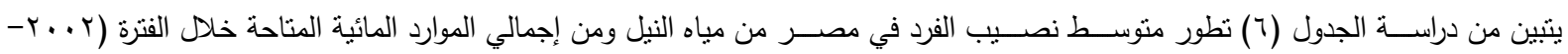

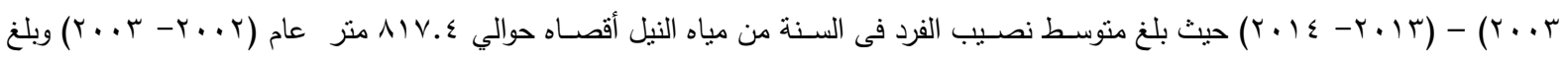

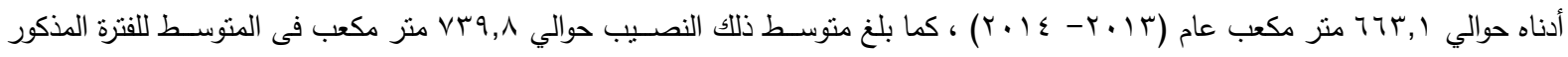

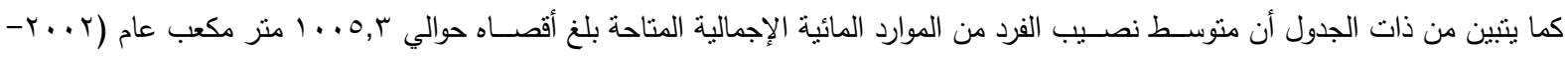

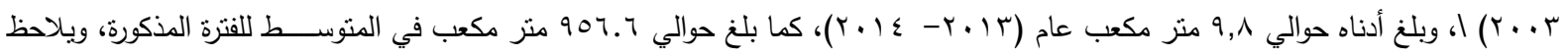

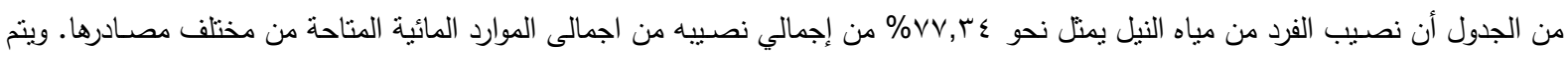
إستيفائه لاحتياجاته من باقي الموارد المائية غير النيلية المناحة.

\begin{tabular}{|c|c|c|c|c|c|}
\hline \multicolumn{2}{|c|}{ متوسط نصيب الفرد من } & \multirow{2}{*}{ 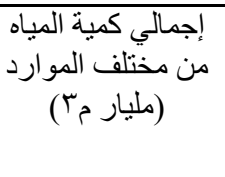 } & \multirow{2}{*}{ (مليار مجّاه } & \multirow{2}{*}{ 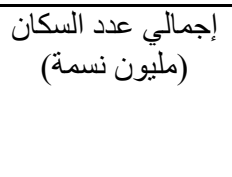 } & \multirow[t]{2}{*}{ بيان } \\
\hline 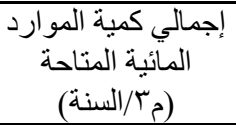 & حصة مصر من مياه النيلة & & & & \\
\hline $1 \cdots 0, r$ & $\Lambda \backslash \vee, \Gamma \wedge$ & $7 \lambda, Y \uparrow$ & 00,0 & $7 V, q$ & $r \ldots r / r \ldots 1$ \\
\hline $99 \cdot, 9$. & $\wedge \cdots, \wedge V$ & $T \lambda, T V$ & 00,0 & $79, r$ & $r \ldots \varepsilon / T \ldots r$ \\
\hline qY^,YY & V^o,, 1 & 79,17 & 00,0 & $V \cdot, V$ & $Y_{\ldots O / T \ldots \varepsilon}$ \\
\hline $97 r, \varepsilon r$ & $\vee \vee \neg \wedge, \vee \cdot$ & 79,07 & 00,0 & VY,Y & $r \ldots T / r \ldots o$ \\
\hline $90 \cdot, 0 \leqslant$ & $v 0 \varepsilon, 1$ & 79,97 & 00,0 & $V T, T$ & $r \ldots V / T \ldots T$ \\
\hline $9 Y Y, 01$ & $V 0 \leqslant, 9 V$ & $V Y, T_{T}$ & 00,0 & $V \varepsilon, \varepsilon$ & $r \ldots N / T \cdots V$ \\
\hline $97 \%, 10$ & $V r_{q}, r$. & $V T, T$. & 00,0 & $v^{7}, 1$ & $r \ldots q / r \ldots 1$ \\
\hline 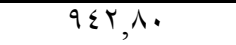 & VIT,TV & VT, ro & 00,0 & $V V, \Lambda$ & $r \cdot 1 \cdot / r \ldots q$ \\
\hline $9 \leqslant Y, \wedge$. & $V I T, T V$ & VT, ro & 00,0 & $V v, \wedge$ & $r \cdot 11 / T \cdot 1$. \\
\hline 941,77 & $79 V, Y \leq$ & $V \varepsilon, 17$ & 00,0 & $\sqrt{9,7}$ & $r \cdot 1 r / r \cdot 11$ \\
\hline $940, Y_{0}$ & $71 \cdot, 10$ & $v_{0,0}$ & 00,0 & 11,7 & $T \cdot I T / T \cdot 1 T$ \\
\hline $9 \cdot 1,1$ & $7 \pi r, \cdot 1$ & 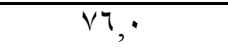 & 00,0 & Ar,, $\mathrm{V}$ & $T \cdot I \leq / T \cdot 1 T$ \\
\hline 907,00 & $V r q, v q$ & $V 1,99$ & 00,0 & vo.rq & المتوسط \\
\hline
\end{tabular}

المصدر: الجهاز المركزي للتعبئة العامة والإحصاء. 
اســتهذف البحث دراســـة الوضـــع الراهن للموارد المائية النيلية والاجمالية المتاحة من مختلف مصـــادرها، ومدى تغطية تللك الموارد

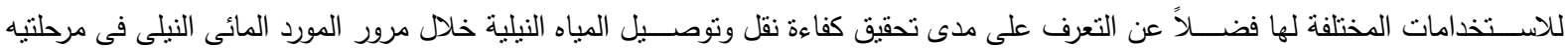
(أسـوان - أفمام الترع)، (أفمام الترع - الحقل)، فضـلاً عن التعرف على نسبة الفوقد المائية خلاد هاتين المرحلتين على مسـتوى مناطق الجمهورية (وجه بحرى، مصر الوسطى، مصر العليا) والتعرف كذلك على نصيب الفرد من المورد المائى النيلى وكذلك من إجمالى الموارد المائية المتاحة من

مختلف مصادرها.

توصلت الدراسة للنتائج التالية:

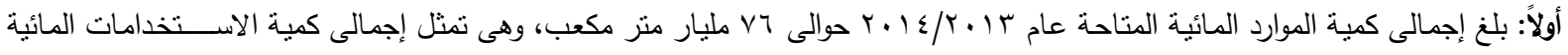

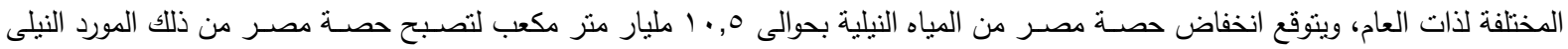
حوالى 0,0 ؛ مليار منز مكعب •

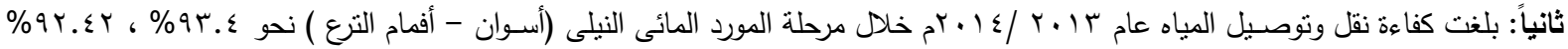

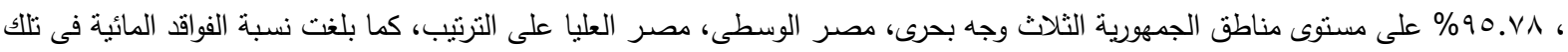

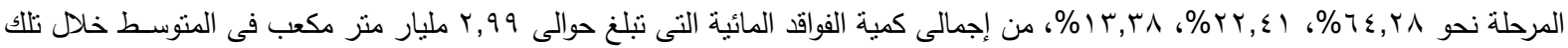

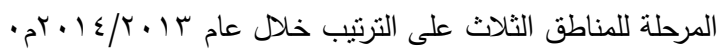

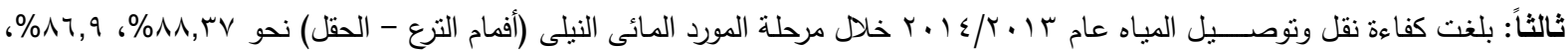

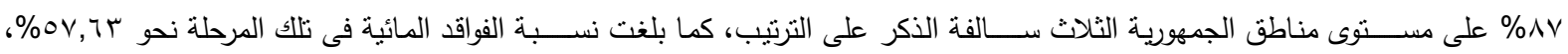

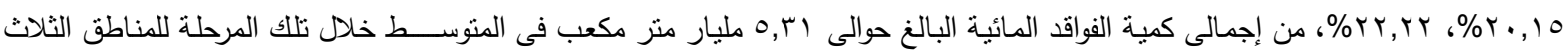
على الترتيب.

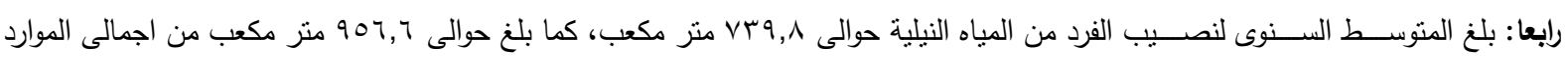

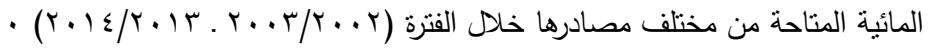

المراجع:

ا. أحمد قدرى مختـار محمد بهلول (دكتور): آثنار علاقات دول حوض نهر النيل على الأمن المـائى المصــري، المؤتمر الثامن عثـــر

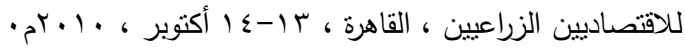

r. أســـامه محمود عويضـــة، الســيد الســـي جاد عبد الرحمن ( دكتوران) الطلب على الموارد المائية في القطاع الزراعى فى ظل المتغيرات

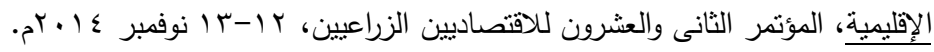

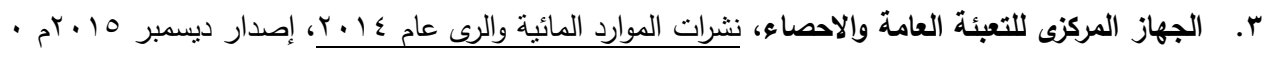
ع. ـ الجهاز المركزى للتعبئة العامة والاحصاء، النشرات السنوية لاحصاءات الرى والموارد المائية، أعداد متفرقة.

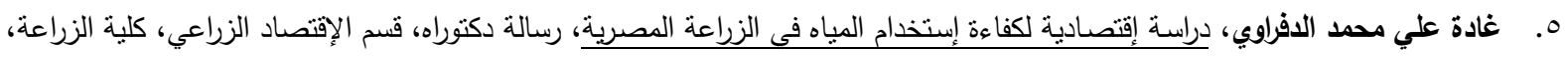

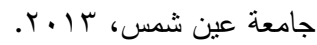
7. محمد صـلاح الدين الجندي (دكتور) وآخرون، تحليل قياسـى لكفاءة إبـتخدام الموارد الزراعية ودورها في مواجهة التحديات التى تواجه القطاع

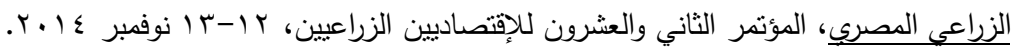

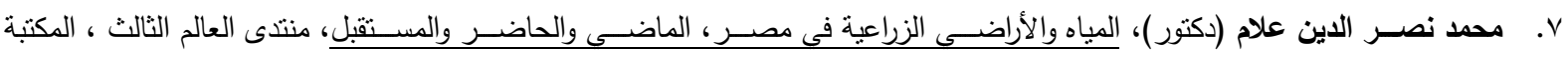

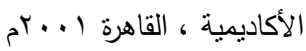

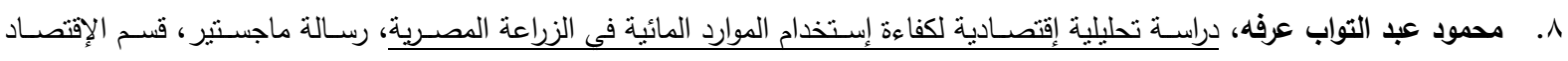

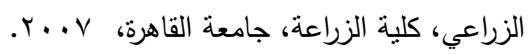




\title{
An economic study for present water situation in Egypt
}

\author{
Prof. Dr. Khairy Taha Ibrahim \\ Emeritus Prof. of Agric. Econ. Faculty of \\ Agriculture Minia University
}

Prof. Dr. Abd El-Naby Abd El-Halim El-Sherief Emeritus Prof. of Agric. Econ. Faculty of Agriculture Al-Azhar University, Assiut

Abo Rehab Esmat Fouad Abo Rehab

Bachelor of Agric. Science (Agric. Econ.)

Faculty of Agriculture, Al-Azhar University, Assiut

\begin{abstract}
Summary
The study aimed at recognizing the present situation for both Nile water resource and total water from different resources, and recognizing direct different water uses in national manner to achieve the maximum satisfaction possible of water in studying of the available supply of water, that amounted to about 76 billion cubic meters, in future decline of about 10.5 billion cubic meters after Ethiopian Renaissance Dam had been built.

The main results in this study as follow:

1- The available supply of water, that amounted about 76 billion cubic meter, in future decline of about 10.5 billion cubic meter after Ethiopian Renaissance Dam had been built.

2- Efficiency transporting of Nile water in (Aswan-on Kanals) stage for three regions (lower Egypt, middle Egypt and upper Egypt) is amounted about 93.2\%, 92.42\%, 95.78\%, respectively. And water losses in this stage amounted about $64.21 \%, 22.41 \%, 13.38 \%$ of total water losses that amounted to 2.99 billion cubic meter in average for the three regions that mentioned, respectively.

3- Efficiency transporting of Nile water in (on canals-fields) stage for three region, (lower Egypt, middle Egypt and upper Egypt) is amounted about $88.37 \%, 86.9 \%, 87 \%$, respectively, And water losses in this stage amounted about $57.63 \%, 20.15 \%, 22.22 \%$ of total water losses that amounted to 5.31 billion cubic meter in average for the three regions, respectively.

4- Annual average per capita from Nile water amounted to 739.8 cubic meter, and 956.6 cubic meter from total different available supply of water, through period (2002/2003 - 2013/2014).
\end{abstract}


